\title{
ACCELERATOR COMPLEX FOR THE JOINT PROJECT OF KEK/JHF AND JAERI/NSP
}

\author{
Y. Yamazaki, M. Mizumoto, and JAERI/KEK Joint Accelerator Team \\ KEK, High Energy Accelerator Research Organization \\ 1-1 Oho, Tsukuba-shi, Ibaraki-ken, 305-0801, Japan \\ JAERI, Japan Atomic Energy Research Institute \\ Tokai-mura, Naka-gun, Ibaraki-ken, 319-1195, Japan
}

\begin{abstract}
The JHF of KEK and the NSP of JAERI were joined to form one project in order to more effectively promote a wide range of scientific and engineering fields included in either of the projects. The Joint Project is divided into two phases. Phase I comprises a 400-MeV linac, a 3-GeV, 1MW rapid-cycling synchrotron and a $50-\mathrm{GeV}$ synchrotron. Phase II is the upgraded system, which includes a several-MW pulsed spallation neutron source. The high-energy linac of the Phase I will be a superconducting one, in order to develop one of the most crucial accelerator techniques for realizing an acceleratordriven nuclear waste transmutation system. The R\&D results accomplished for the Joint Project are highlighted.
\end{abstract}

\section{INTRODUCTION}

High Energy Accelerator Research Organization (KEK) and Japan Atomic Energy Research Institute (JAERI) agreed with each other to bring the Japan Hadron Facility (JHF) Project [1-7] of KEK and the Neutron Science Project (NSP) [8-10] of JAERI into one joint project. Both projects have some common features which can be summarized by a single key word, that is, "high-power proton accelerators." The JHF project was from the beginning a joint one of the former KEK and the former Institute of Nuclear Study (INS) of University of Tokyo, which are joined together to form the present KEK in order to realize the JHF. These results evidently show how a wide variety of science and engineering fields are requiring high-power proton accelerators.

It was planned that the JHF comprises a $50-\mathrm{GeV}$ synchrotron, a $3-\mathrm{GeV}$ rapid-cycling synchrotron (RCS), and a 200-MeV linac [1-7]. The slowly extracted 50-GeV beam is used for both fundamental particle physics and nuclear physics, such as Kaon rare decay and hypernuclei spectroscopy. The fast-extracted one is used for a longbaseline tau-neutrino appearance experiment, by fully utilizing the SUPERKAMIOKANDE detector located $250 \mathrm{~km}$ from KEK. The $3-\mathrm{GeV}$ beam is to be fastextracted to three experimental areas: a pulsed spallationneutron experimental area, a muon experimental area, and an exotic nuclei experimental area. In this way the JHF project would cover fundamental particle physics, nuclear physics, materials science, life science, and others. The project has been promoted by the related scientific communities as a first-priority project.
In contrast to the basic science nature of the JHF project, NSP has been oriented to engineering and industrial fields as well as basic science. It is aiming at higher proton beam power, based upon the high-intensity proton linac. Originally proposed for the acceleratordriven nuclear waste transmutation system(ADTS or ADS), that is, as one of the nuclear energy project OMEGA[11,12], the JAERI high-power proton project has gradually been shifted to science using a spallation neutron source. Since the ADS is also making use of spallation neutrons, this project is referred to as the "Neutron Science Project". The accelerator complex of the present NSP comprises a $1.5-\mathrm{GeV}$ proton linac and storage rings (SR's). Since the ADS ultimately requires CW operation, the linac should be compatible with both CW operation and pulse operation. A superconducting (SC) linac is a natural choice for CW operation [13,14].

Both the JHF project and NSP include pulsed spallation neutron sources. The former one is of rather moderate power (0.6 MW), while the latter is ambitiously aiming at an extremely high power of up to $5 \mathrm{MW}$. The neutronscience community in Japan has assigned the former as the present, most urgent project, considering the latter as a future one. Meanwhile, JHF was slightly modified in order to assure a future upgrade to a 1-MW class machine. On the other hand, the 5-MW neutron source is very difficult to achieve, requiring intensive development. In this way, we consider it ideal to properly join the two projects from both the viewpoints of the usage and developments of high-power proton accelerators.

The Joint Project now covers both basic science and the engineering, that is, nuclear-energy applications. This is the natural result of the recent widening of the application fields of the high-power proton accelerators.

In the following section, we discuss the accelerator scheme of the Joint Project and our strategy for developing and realizing extremely high-power proton accelerators. Then, the research and development work will be presented, as has been done for the JHF and NSP, and will be elucidated from the new viewpoint of a Joint Project. In particular, we will show how the innovative results of the $\mathrm{R} \& \mathrm{D}$ have influenced us in order to formulate the above-mentioned strategy. 


\section{ACCELERATOR SCHEME OF JOINT PROJECT AND ITS USAGE}

The accelerator complex of the Joint Project will be built at the Tokai site of JAERI, shown in Fig. 1. The project is divided into two phases. Phase I includes:

1) a $50-\mathrm{GeV}$ main synchrotron with an average current of $15 \mu \mathrm{A}$ and a repetition rate of $0.3 \mathrm{~Hz}$,

2) a 3-GeV RCS with an average current of $333 \mu \mathrm{A}$ and a repetition rate of $25 \mathrm{~Hz}$, and

3) a $400-\mathrm{MeV}$ proton linac with a peak current of $50 \mathrm{~mA}$, a pulse length of $500 \mu \mathrm{s}$, and a repetition of $25 \mathrm{~Hz}$.

Both the RCS and linac should be upgradable regarding their energy and intensity.

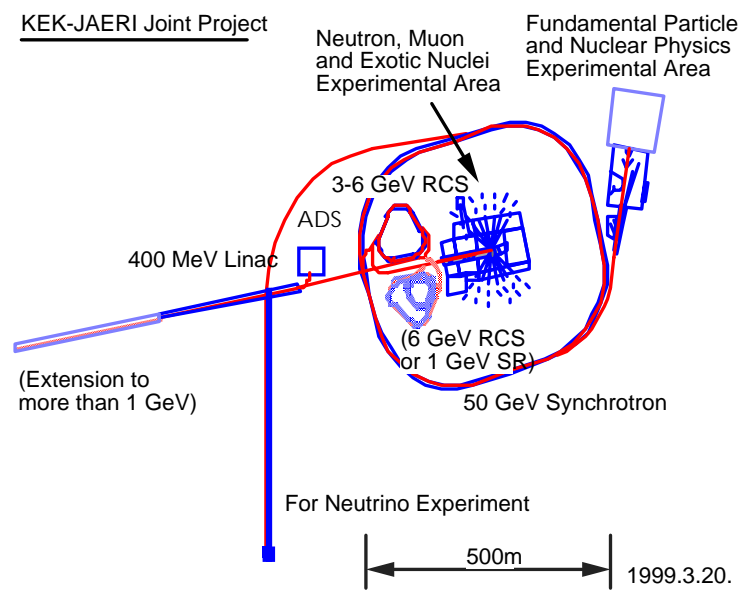

Fig. 1 Layout of the accelerator complex of the Joint Project. The facilities with parentheses are for the future upgrade.

The use of the $50-\mathrm{GeV}$ synchrotron is exactly the same as in the JHF project. On the other hand, the $3-\mathrm{GeV}, 1-$ MW beam will be provided to the pulsed spallation neutron experiment in the same way as both the JHF project and NSP. It will also be used for muon science, the production target for which is located upstream of the neutron source as well as that for exotic nuclei science.

A unique feature of the joint project is, from the user's view point, that the long-pulse, $400-\mathrm{MeV}$ beam from the linac is transported to the ADS experimental area. From the accelerator viewpoint regarding ADS, the high-energy part of the $400-\mathrm{MeV}$ linac will use SC accelerating cavities, which can be a prototype of the future $\mathrm{CW}$ accelerator for ADS use. The development work for the SC proton linac has been performed at JAERI [8-10] in collaboration with the SC linear collider group at KEK [15] as described in Sec. 3.3. In this way, the ADS technology will be steadily developed regarding both the target side and the accelerator side.

Phase II of the Joint Project is of course the upgrade of the Phase I. Another unique feature of the Joint Project is the high compatibility with various upgrade paths of the accelerator complex as follows. It is controversial which accelerator scheme is more realistic[13], the scheme of a full-energy linac with SR's or the RCS scheme, in order to achieve a several-MW pulsed neutron source. One of the major limiting factors on high-power proton accelerators is radioactivity arising from beam loss. In particular, beam loss is unavoidable, and most serious during the injection process to a ring, whether it is an SR or an RCS. Since the injection energy to the RCS is significantly lower than that to the SR, the radioactivity can be lowered by approximately the ratio of the former injection energy to the latter one. In addition, since the accelerated beam energy of the RCS can be higher than that of the SR, we can lower the beam current of the RCS more than that of the SR with the same power, that is, approximately the same neutron yield. In this two-folding way, we can allow a higher beam loss rate in the RCS scheme than that in the SR scheme, assuming the same neutron flux and the same allowable radioactivity (approximately a factor of 7.5 in the Phase I). It is true that the SR's are more advantageous than RCS regarding many features. For example, the high-power RF system required for a high-energy RCS is much more complicated than that for an SR. However, it should be noted that the recent innovative work done for the JHFring RF system [16-18] has significantly eased one of the major problems existing in the high-energy RCS, as detailed in Sec. 3.1. This kind of innovative work may change the pros and cons of competitive options. In any case, however, a beam study is indispensable in order to critically test which problems existing in the RCS scheme or the SR scheme are more serious.

The Phase-I machines will be fully utilized for this test. The several-GeV booster synchrotron is indispensable for the high-intensity several $10-\mathrm{GeV}$ beams. This is one of the reasons why we are going to use the RCS for the Phase-I neutron source in contrast to the SNS project [19] and the ESS project [20]. If it turns out that the RCS scheme is more promising than the SR scheme, we will upgrade the Phase-I RCS to $6 \mathrm{GeV}$ and will build one more 6-GeV RCS in order to provide a several-MW beam. In contrast, if the result is opposite, we will upgrade the linac to more than $1 \mathrm{GeV}$ and will construct two or three SR's. Either path is possible, as seen from Fig. 1. The Phase-I linac will be constructed at a location where an energy upgrade is possible, if necessary. The upgrade paths conceived in the Joint Project are summarized in Table 1. The optimum may be in between the two extreme options.

Being logically independent of the pulsed spallation neutron science (not financially independent), further ADS development may require a higher linac energy. In that case, the linac energy will be upgraded in the same way as in the SR scheme for the neutron science. However, it should be here emphasized that the severalMW neutron source is too difficult to realize by any compromised scheme. It can only be realized for its own purpose. 
Table 1. Upgrade Path of Linac and Rings

\begin{tabular}{|c|c|c|c|c|c|c|c|c|}
\hline & \multicolumn{3}{|c|}{ RCS or SR } & & \multicolumn{4}{|c|}{ Linac (56 \% chopping) } \\
\hline $\begin{array}{l}\text { Beam } \\
\text { Power }\end{array}$ & Energy & Repetition & Number & Energy & $\begin{array}{l}\text { Peak } \\
\text { Current }\end{array}$ & $\begin{array}{l}\text { Pulse } \\
\text { Length }\end{array}$ & Repetition & $\begin{array}{l}\text { Average } \\
\text { Current }\end{array}$ \\
\hline $1.0 \mathrm{MW}$ & $3 \mathrm{GeV}$ & $25 \mathrm{~Hz}$ & 1 & $400 \mathrm{MeV}$ & $50 \mathrm{~mA}$ & $500 \mu \mathrm{s}$ & $25 \mathrm{~Hz}$ & $333 \mu \mathrm{A}$ \\
\hline $.0 \mathrm{MW}$ & $6 \mathrm{GeV}$ & $25 \mathrm{~Hz}$ & 2 & $400 \mathrm{MeV}$ & $60 \mathrm{~mA}$ & $500 \mu \mathrm{s}$ & $50 \mathrm{~Hz}$ & $833 \mu \mathrm{A}$ \\
\hline $5.0 \mathrm{MW}$ & $1 \mathrm{GeV}$ & $50 \mathrm{~Hz}$ & 2 or 3 & $1 \mathrm{GeV}$ & $60 \mathrm{~mA}$ & $3 \mathrm{~ms}$ & $50 \mathrm{~Hz}$ & $5 \mathrm{~mA}$ \\
\hline
\end{tabular}

a) The flat bottom of the RCS magnet power supply is lengthened.

The average current is obtained by a product of the peak current, the pulse length, the repetition, and the chopping rate of $56 \%$.

Construction of the $60-\mathrm{MeV}$ part of the linac was already started in KEK as the low-energy front of the $200-\mathrm{MeV}$ linac $[2,5,6]$ for the JHF project, which comprises a 3-MeV, 324-MHz RFQ linac, a 50-MeV, 324-MHz drift-tube linac (DTL), and a $200-\mathrm{MeV}, 324-$ $\mathrm{MHz}$ Separated DTL (SDTL). The beam will be commissioned by the end of 2000. This part will be transported to the JAERI/Tokai site in order to fully use it for the Joint Project.

$\mathrm{H}^{\text {}}$ ions with a peak current of $50 \mathrm{~mA}$ are accelerated up to $400 \mathrm{MeV}$ by the linac. Then, the ions are injected to the 3-GeV RCS through a piece of charge-exchange foil. The large acceptance in the ring is transversely painted in order to maximally ease the space-charge effect. (The longitudinal painting will be done not intentionally, but partly by the sinusoidally varying magnetic fields.) The beam has already been chopped in the linac synchronously with $1.3-\mathrm{MHz} \mathrm{RF}$ acceleration in the RCS in order to longitudinally accept all beams from the linac. In this way, we can avoid the beam loss which is inherent in adiabatic capture. Two buckets are thus filled out for $500 \mu \mathrm{s}$.

The magnetic fields are to sinusoidally oscillate with a rapid cycle of $25 \mathrm{~Hz}$. Each of the focusing quadrupoles, defocusing quadrupoles and bending magnets is to be driven through its own resonant network in order to maintain a greater number of adjustable knobs. The injection time is to be limited by the approximately flat bottom of the $25-\mathrm{Hz}$ sinusoidal function. In each cycle the ring will accelerate $8 \cdot 10^{13}$ protons.

The two bunches thus accelerated are to be injected four times to the $50-\mathrm{GeV}$ synchrotron, in which eight bunches are accelerated $\left(3.2 \cdot 10^{14} \mathrm{ppp}\right)$. Two buckets are left empty in order to allow the rising of the fastextraction kickers during the time thus opened. After the 0.12 -s injection the beam energy is to be ramped up to 50 $\mathrm{GeV}$ for $1.9 \mathrm{~s}$, and then the beam will be slowly extracted during a time of $0.7 \mathrm{~s}$. The ramping cycle is to be completed in $3.42 \mathrm{~s}$, including the falling time of $0.7 \mathrm{~s}$.

\section{R \& D RESULTS FOR THE JOINT PROJECT}

The R\&D programs were formed for the JHF project and NSP in order to overcome various difficulties associated with their high-intensity character. The results so far accomplished are highlighted below.

\section{1 Accelerating cavities loaded with magnet alloy}

It has been seen that the RCS for the joint project is characterized by a challengingly high energy and rapid cycling, even for Phase I. The rapid acceleration required for this option is very difficult to obtain by using the conventional ferrite-loaded cavities, in particular under the heavy beam loading. Although the ferrite material has been widely used for proton synchrotrons, because of its excellent permeability and tunability, it is still suffering from the following difficulties:

1) Since its saturation field is rather low, its $\mu \mathrm{Qf}$ value, which is proportional to the shunt impedance, rapidly decreases as the operating magnetic field (thus, the field gradient) is increased. Together with its rather low Curie temperature (typically $100^{\circ} \mathrm{C}$ to $200^{\circ} \mathrm{C}$ ), the highestpossible field gradient is limited to approximately 20 $\mathrm{kV} / \mathrm{m}$.

2) In order to tune a ferrite-loaded cavity by adjusting its permeability, it is necessary to supply the DC magnetic field on the ferrite through a bias circuit. The resulting complicated RF system viewed from the beams makes it difficult to analyze in order to overcome heavy beamloading problems. Even worse, the response to the bias current is sometimes too slow for rapid cycling.

3) The high $Q$ value, typically around a few ten, gives rise to coupled-bunch instabilities, which should be cured for high-current operation.

In particular, the problem 1) is most serious, as can be understood by attempting a lattice design for the highenergy RCS. Many long straight sections have to be prepared for the rapid acceleration, thus increasing the machine cost significantly.

Magnetic alloys (MA's), for example, FINEMET, appear to be very promising to simultaneously solve the above-mentioned problems [16-18]. The $\mu \mathrm{Qf}$ value of MA's is almost constant throughout the wide range of the magnetic field valuesin contrast to that of ferrites. Together with its high Curie temperature (typically of $570^{\circ} \mathrm{C}$ ) and easy-cooling taping structure, the former appears to be so promising for obtaining a high field gradient. Furthermore, in spite of its high $\mu \mathrm{Qf}$ value, the 
Q value is extremely low, typically around unity. Consequently, no tuning is necessary for the MA-loaded cavities.

In order to demonstrate the real usefulness of the MAloaded cavities, they have been power-tested [16] and beam-tested by electron beams [16]. One of the prototypes was actually used for beam acceleration in the HIMAC synchrotron [16,17]. The other cavity loaded with water-cooled MA plates was powered up to $50 \mathrm{kV} / \mathrm{m}$ [16], which is several times as high as the conventional ferrite-loaded cavity. The tested field gradient was limited by the RF power rather than the MA performance.

The extremely low $Q$ value of an MA-loaded cavity makes it ideal for the barrier-bucket application. Another prototype cavity was shipped, and installed to the BNL/AGS under the Japan/US high-energy physics collaboration program. The barrier bucket was generated in the AGS together with the BNL cavity [18]. The beamloading compensation has been easily done by the oneturn feed-forward method[16], since the cavity is like a pure resistance rather than the resonant circuit.

On the basis of the above empirical test results, we decided to use MA-loaded cavities for both the RCS and $50-\mathrm{GeV}$ synchrotron. It is noted that they can be used not only for acceleration, but also for barrier-bucket generation and for second-harmonic cavities. The latter two applications will greatly ease both the space-charge problem and longitudinal instability by improving the bunching factor.

\section{2 Low- and Medium-Energy Linac}

These days it becomes common to use an RFQ with a rather high energy. This trend was initiated by the 3$\mathrm{MeV}, 432-\mathrm{MHz}$ RFQ linac [21], which was realized by inventing the $\pi$-mode stabilizing loop (PISL) [22]. This RFQ successfully accelerated a $\mathrm{H}$ beam of $13 \mathrm{~mA}$ peak [21].

In contrast to the medium duty of a few percent for the JHF, the very high duty of a few ten percent was a main challenge of the R\&D program for the NSP. The $2-\mathrm{MeV}$, 200-MHz RFQ linac was tested for a proton peak current of $70 \mathrm{~mA}$ with a duty factor of $10 \%$ (a beam pulse length of $1 \mathrm{~ms}$ and a repetition of $100 \mathrm{~Hz}$ ) [23]. A peak current of $100 \mathrm{~mA}$ was achieved at the duty factor of $1 \%$ (a beam pulse length of $1 \mathrm{~ms}$ and a repetition of $10 \mathrm{~Hz}$ ). The first 9-cell of the DTL was power-tested up to the duty factor of $20 \%$ and $50 \%$ with the field gradients of $2 \mathrm{MV} / \mathrm{m}$ and $1.7 \mathrm{MV} / \mathrm{m}$, respectively. The joint effort will realize highly stable RFQ and DTL with a high duty of $15 \%$ for the Phase II.

\section{3 High-Energy Linac}

The SC cavities for the proton linac have been intensively developed by the JAERI/NSP group [10] in close collaboration with the SC linear collider group [15] in KEK. Among various accomplishments of this R\&D program, it is emphasized that the world-highest accelerating field gradient of $8.8 \mathrm{MV} / \mathrm{m}$ (the maximum surface field of $44 \mathrm{MV} / \mathrm{m}$ ) has been obtained for a $\beta=0.5$ structure [10]. In particular, the electropolishing technique [15] developed and mass-used for the KEK/TRISTAN was fully utilized for this accomplishment.

It is true that the pulse-mode operation of the SC cavities loses one of the most important advantages regarding energy savings [13]. However, the pulse length for the Joint Project is sufficiently long for making the SC and normal-conducting (NC) options competing. Then, the other advantages of the SC option are appreciated [13]: the higher field gradient, the higher stored energy and the larger bore radius. We consider that the reliability problem so far existing in the SC technology has been mostly solved by recent intensive efforts, including the above development. In particular, the recent development of the high-power input coupler for the SC cavities should be emphasized [24].

The most serious issue is how to overcome the problems associated with the pulse operation of the SC cavities, such as microphonic vibration problem and phase/amplitude control of the accelerating field. The extremely high accuracy required for the phase/amplitude control in the high-power proton linac in comparison with that for electron accelerators is very difficult to obtain for the pulse operation under the heavy beam loading.

The annular-ring coupled structure (ACS) first realized in KEK $[25,26]$ was ready for normal-conducting back-up option. The ACS is characterized by the balanced performance: its shunt impedance and coupling are comparable to those of the widely-used side-coupled structure, while it has an axially symmetry which may be important regarding the minimization of halo formation [26].

\section{3 Other Accomplishments}

In addition to the above accomplishments, the linac group has been developing the following items:

1) A volume-production type $\mathrm{H}^{-}$ion source, which generates a peak current of $16 \mathrm{~mA}$ with a normalized $90 \%$ emittance of $0.5 \pi \mathrm{mm}$ mrad without cesium [21]. For the NSP linac a $\mathrm{H}^{-}$ion peak current of $21 \mathrm{~mA}$ was obtained with a current density of $33 \mathrm{~mA} / \mathrm{cm}^{2}$ and a duty factor of $5 \%$ with Cesium seeded [27].

2) A newly optimized low-energy beam transport (LEBT) made of two solenoids [21]. The optimization work was based upon careful three-dimensional analyses of various LEBT schemes, while carefully taking into account the geometrical aberration. The beam experiment showed that a very strong space-charge neutralization effect maintained the high brightness throughout the LEBT.

3) Separated DTL (SDTL) [28] after around $50 \mathrm{MeV}$. Its idea is based upon the fact that quadrupole magnets (QM's) are not necessary in every drift tube (DT) after 50 $\mathrm{MeV}$. By taking the QM's outside the DT's, that is, outside of the tank, we can optimize the geometrical shapes of the DT's in order to maximize the shunt impedance. The shunt impedance of SDTL is by 40 to 65 percent higher than that of a conventional DTL with the same frequency.

4) RF chopper after the 3-MeV RFQ. The chopping is one of the most difficult items to be developed. At present a 
chopping system has been designed which is compatible with a beam loss of 1 percent in the linac [29].

The ring group has been developing the following items:

1) Transition-free lattice for the $50-\mathrm{GeV}$ main synchrotron [3],

2) $50-\mathrm{GeV}$ synchrotron magnet power supplies $[2,3]$,

3) RCS magnet power supplies [2], and

4) ceramics vacuum chambers for the RCS.

The imaginary transition $\gamma\left(\gamma_{t}\right)$, that is, negativemomentum compaction factor $(\alpha)$, should be realized in order to make the ring free of the transition. The imaginary $\gamma_{t}$ is a unique feature of the lattice for the 50$\mathrm{GeV}$ main ring. The synchrotron oscillation, the frequency of which is proportional to the square root of the slippage factor $(\eta)$, loses stability at $\eta=0$, resulting in beam loss. The beam energy and the value of $\gamma$ which make $\eta$ vanish are referred to as the transition energy and transition $\gamma\left(\gamma_{t}\right)$, respectively. Since $\alpha$ is proportional to an orbit integral of the dispersion function divided by the bending radius $(\rho)$, we can obtain a negative $\alpha$ if the dispersion function can be made negative at some of the bending magnets. Among various methods, we have chosen the missing-bend method rather than beta-function modulation, which is fairly harmful in any case.

The magnet power supplies of the $50-\mathrm{GeV}$ synchrotron will use insulated gate bipolar transistors (IGBT), the gating time of which is so fast and flexible as to avoid a harmful reactive (wattles) power. This is also a longwaited device, but it is only recent that high-power devices (3.3 kV, $1200 \mathrm{~A})$ have been successfully developed. Since the development is still continuing, we are expecting that the devices can be mass-used for our power supplies.

Three families of the magnets of the RCS respectively driven through three resonant networks need precise amplitude and phase controls in order to synchronously operate three systems. Two prototypes of the resonant networks have been fabricated and tested. They were successfully in phase within $1 \mathrm{mrad}$, which corresponds to 0.01 in the betatron tune difference.

The rapid cycle of $25 \mathrm{~Hz}$ of the RCS led us to use a ceramics chamber in order to avoid any harmful effect of the eddy current otherwise induced. The chamber, on the other hand, should RF-shield the beam current by means of copper strips or copper plating, or other. The R\&D including the fabrication of the ceramics chamber is in progress.

\section{CONCLUSION}

The high-power proton accelerator project in Japan is strongly supported not only by the neutron-science community, but also by many scientific communities. In addition, the ADS is another engineering and industrial field to seriously develop. The Joint Project will effectively cover all of these requirements, thus making itself a very unique project, internationally supported by nuclear physicists and fundamental particle physicists.
On the other hand the user's request stimulated the development of the accelerator technology in such a way that the SC linac feasibility is seriously investigated for the ADS technology. Another example is the RCS, which plays both roles of a booster to the higher-energy synchrotron and a pulsed spallation neutron source. The request for the high-energy, high-intensity RCS stimulated the accelerator group to invent a new type of accelerating cavities. In this way, the Joint Project becomes unique also from the viewpoint of accelerator technology. It will present an accelerator test bench in order to determine which is more promising, RCS or SR, for higher power proton accelerators.

\section{REFERENCES}

[1] JHF Project Office, "Proposal for Japan Hadron Facility," KEK Report 97-3 (JHF-97-1).

[2] JHF Project Office, "JHF Accelerator Design Study Report" KEK Report 97-16 (JHF-97-10).

[3] Y. Mori, Proc. 1997 Part. Accel.Conf., 920 (1997).

[4] Y. Yamazaki, Proc. 1st Asian Part. Accel. Conf., 5A002-314 (1998).

[5] T. Kato, KEK Report 96-17 (1997).

[6] Y. Yamazaki and T. Kato, Proc. 1998 Linac Conf., TU4011 (1998).

[7] Y. Mori, Proc. 1st Asian Part. Accel. Conf., 5D001-375 (1998)

[8] M. Mizumoto et al., Proc. 1st Asian Part. Accel. Conf., 5A001309 (1998).

[9] M. Mizumoto et al., "A High Intensity Proton Linac Development for the JAERI Neutron Science Project", Proc. 1998 Linac Conf., TU1004 (1998)

[10] N. Ouchi et al., "Development of Superconducting Cavities for High Intensity Proton Accelerator at JAERI", Proc. 1998 Applied Superconducting Conf. (1998).

[11] Y. Kaneko, "The Intense Proton Acclerator Program, " Proc. the 2nd International Symposium on Advanced Nuclear Energy Research, Mito, p. 25 (1990).

[12] M. Mizumoto, Proc. 1994 Linac Conf., 317 (1994)

[13] Y. Yamazaki, Proc. 1996 Linac Conf., 592 (1996).

[14] Y. Yamazaki, " Accelerator Development for the Japanese Hadron Project and Continuous-Beam, Superconducting Proton Linac", Proc. Workshop for JAERI Proton Technology Center Project, KEK Preprint 95-64 (1995) (in Japanese).

[15] E. Kako et al., "Improvement of Cavity Performance by Electropolishing in the $1.3 \mathrm{GHz} \mathrm{Nb}$ Superconducting Cavities", this conference, THAL6.

[16] C. Ohmori et al., "High Field-Gradient Cavities Loaded with Magnetic Alloys", this conference, THAL1.

[17] R. Muramatsu et al., "The First Beam Acceleration Test using High Gradient Cavity at HIMAC", this conference, MOP59.

[18] M. Fujieda et al., "Magnetic Alloy Loaded RF Cavity for Barrier Bucket Experiment at the AGS", this conference, MOP81.

[19] J. Alonso, "The Spallation Neutron Source Program", this conference, FRA01.

[20] K. Bongardt et al., Proc. 5th European Part. Accel. Conf., 158 (1996).

[21] A. Ueno et al., Proc. 1996 Linac Conf., 293 (1996)

[22] A. Ueno and Y. Yamazaki, Nucl. Instr. Meth. A300, 15 (1990).

[23] K. Hasegawa et al., "Development of a High Intensity RFQ at JAERI", Journal of Nuclear Science and Technology, 34, 622 (1997).

[24] S. Mitsunobu et al., "High Power Test of the Input Coupler for KEKB SC Cavity", Proc. 7th Workshop on RF Superconductivity, 735 (1995).

[25] T. Kageyama et al., Part. Accel. 32, 33(1990)

[26] T. Kageyama et al., Proc. 1992 Linac Conf., 456 (1992)

[27] H. Oguri et al., "Development of an Injector Section for the High Intensity Proton Accelerator at JAERI", Proc. 1998 European Part. Accel. Conf., (1998).

[28] T. Kato, KEK Report 92-10 (1992).

[29] T. Kato and S. Fu, "MEBT Design for the JHF 200-MeV Proton Linac", Proc. 1998 Linac Conf., MO4012 (1998). 